Nöbeling G., [1] Topologie der Vereine und Verbände. Archiv der Mathematik 1 (1949), pp. 354-159.

Saks S., [1] Zarys teorii calki. Warszawa 1930.

Sikorski R., [1] On the representation of Boolean algebras as fields of sets. Fund. Math. 35 (1948), pp. 247-258.

- [2] On the inducing of homomorphisms by mappings. Fund. Math. this volume, pp. $\mathrm{i}-22$.

- [3] Sur les corps de Boole topologiques. C. R. Paris 226 (1948) pp. $1675-1676$.

- [4] Sur la convergence des suites d'homomorphies. Ihid., pp. 1792-1793. - [5] On a generalization of theorems of Banach and Cantor-Bernstein. Coll. Math. 1 (1948), pp. 140-144.

- [6] A theorem on extension of homomorphisms. Annales Soc. Pol. Math. 21 (1948), pp. 332-335

Terasaka H., [1] Die Theorie der topologischen Verbände. Reprint of that part of vol. 33 of Fundamenta Mathematicae which was printed in 1939, before the war (pp. 1-33).

\section{On the boundary values of functions of several complex variables, I.}

By

\section{A. Z y g m und (Chicago, U.S.A.).}

1. Introduction. Let $f(\zeta)$ be a function regular for $|\zeta|<1$. Fatou's classical result asserts that if $f(\zeta)$ is bounded there, then for almost every point $\epsilon^{i \theta}$ on $|\zeta|=1$ the limit of $f(\zeta)$ exists as $\zeta$ approaches $\epsilon^{i \theta}$ along any non-tangential path. A more general result of Nevanliuna and Ostrowski asserts that the conclusion of Fatou's theorem holds if the boundedness of the function $f$ is replaced by the condition

$$
\int_{0}^{2 \pi} \log +\left|f\left(\varrho e^{i \theta}\right)\right| d \theta=0(1) .
$$

In a sense, this is the best possible result since, if $\omega(u)$ is any positive and increasing function tending to $+\infty$ with $u$ but less rapidly than $\log u$, then there is a function $f(\zeta)$ regular for $|\zeta|<1$, satisfying the condition $\int_{0}^{2 \pi} \omega\left(\left|f\left(\varrho e^{i \theta}\right)\right|\right) d \theta=0(1)$, and depriced eren of radial limit for almost every $\theta^{1}$ ).

The main problem of this paper is that of boundary ralues of regular functions of several complex variables. Let us begin by the simplest case of two variables, and let $f(z, \zeta)$ be regular in the bicylinder $|z|<1,|\xi|<1$. Thus

$$
f(z, \zeta)=\sum_{0,0}^{\infty} c_{m n} 2^{m} \zeta^{n}
$$

The first question that naturally occurs is whether an analogue of Fatou's theorem holds here. The answer is affirmative: if $f(z, \zeta)$ is bounded for $|z|<1,|\zeta|<1$, then

$$
\lim _{z \rightarrow e^{i x}, \zeta \rightarrow e^{i t}} f(z, \zeta)
$$

1) See Paley and Zygmund [1]. 
exists for almost every point $(x, \theta)$ of the square $0 \leqslant x \leqslant 2 \pi, 0 \leqslant \theta \leqslant 2 \pi$. provided that $\approx$ tends to $\epsilon^{i x}$ alorg any non-targential path and $\zeta$ tends to $t^{i \theta}$ alorg any non-targertial path. (In what follows we shall say for brevity that the poirt $(z, \zeta)$ tends to $\left(\epsilon^{i x}, e^{i \theta}\right)$ along any non-targential path. Similarly for any number of complex. variables) ${ }^{2}$ ). More difficult is the problem of an extension to the case of two variables of the Nevanlinna-Ostrowski theorem. We shall show that the conclusion of the precedirg result holds if the boundedness of $f(z, \zeta)$ for $|z|<1,|\zeta|<1$ is replaced by the condition.

(2) $\left.\int_{0}^{2 \pi} \int_{0}^{2 \pi} \log ^{+} \mid f\left(r \epsilon^{i x}, \varrho \epsilon^{i \theta}\right)\right] \log +\log +\left|f\left(r^{r} \epsilon^{i x}, \varrho \epsilon^{i \theta}\right)\right| d x d \theta<M<+\infty$.

More generally, we shall prove the following theorem.

Theorem 1. Let $f\left(z_{1}, z_{2}, \ldots, z_{k}\right)$ be regular in the k-cylinder

$\left(T_{k}^{r}\right)$ and let the integrat

$$
\left|z_{1}\right|<1, \quad\left|z_{2}\right|<1, \quad \ldots, \quad\left|z_{k}\right|<1
$$

(3) $\int_{0}^{2 \pi} \ldots \int_{0}^{2 \pi} \log +\left|f\left(r_{1} t^{i x_{1}}, \ldots, r_{k} t^{i x_{k}}\right)\right|\left\{\log +\log +\left|f\left(r_{1} \epsilon^{i x_{1}}, \ldots, r_{k} t^{i x_{k}}\right)\right|\right\}^{k-1} d x_{1} \ldots d a_{k}$

be bounded for all $r_{i}<1$. Then, for almost every point $\left(x_{1}, x_{2}, \ldots, x_{k}\right)$ of the $k$-dimensional interval

$\left(Q_{k}\right)$

$$
0 \leqslant x_{1} \leqslant 2 \pi, \quad 0 \leqslant x_{2} \leqslant 2 \pi, \quad \ldots, \quad 0 \leqslant x_{k} \leqslant 2 \pi
$$

the limit $f\left(f^{i x_{1}}, t^{i x_{2}}, \ldots, t^{i x_{k}}\right)$ of $f\left(z_{1}, z_{2}, . ., z_{k}\right)$ exorsts as $\left(z_{1}, z_{2}, \ldots, z_{k}\right)$ approaches $\left(\epsilon^{i x_{1}}, \pm^{i x_{2}}, \ldots, t^{i x_{k}}\right)$ alorg any non-targ ${ }^{2 n t i a l}$ path $\left.{ }^{3}\right)$.

2) See Zygmund [2], where the result is stated explicitly for the radial approach only (as a result abuut Abel summability of double Fourier series), though the proof is essentially the same for non-tangential paths. More precisely among other results, it is shown there that if $\sum_{-\infty}^{+\infty}\left|c_{m n}\right|^{2}$ converges and if we set

$$
u(x, \theta ; r, \varrho)=\sum_{-\infty}^{+\infty} c_{m n} \mid{ }^{|m|} \rho^{|n|} e^{i(m x+n(t)}
$$

then at almost every point $x, \theta$ the expression $u$ tends to a finite limit as $r, \varrho \rightarrow 1$ If $f(z, \zeta)=\sum_{0}^{\infty} c_{m n} z^{m} \zeta^{n}$ is bounded for $|z|<1,|\xi|<1$, then clearly $\sum\left|c_{m n}\right|^{2}<c$. 3) By a limit we always mean a finite limit.
At the first sight, the condition of boundedness of the integral (3) may appear rather-strange. To grasp -its significance, one has to recall a result from the theory of Lebesgue integration, namely that if $g\left(x_{1}, x_{2}, \ldots, x_{k}\right)$ is measurable and $|g|\left(\log +\mid g^{i}\right)^{k-1}$ is integrable, then the integral of $g$ is strongly differentiable at almost every point $\left.{ }^{4}\right)$. The resemblance between these two theorems is by no means coincidental since their common source is the Hardy-Littlewood Max Theorem ${ }^{5}$ ).

One may ask about the behaviour of $f$ if the iterated logarithm in (3) is omitted. To a fuller discussion of this problem we return in the second part of this paper. Here we limit ourselves to a result which will be needed in the proof of Theorem 1. Here and hereafter, unless otherwise stated, by a limit we shall always mean a limit alor $g$ every non-targential path. For the sake of clearness we begin by stating the result for $k=2$.

Theorem $\boldsymbol{2}^{\prime}$. Let $f(z, \zeta)$, regular for $|z|<1,|\zeta|<1$, satisfy the condition

$$
\int_{0}^{2 \pi} \int_{0}^{2 \pi} \log +\left|f\left(r \epsilon^{i x}, \varrho \epsilon^{i \theta}\right)\right| d x d \theta<M<\infty
$$

Then, for almost every $\theta$ in $(0,2 \pi)$ the limit

$$
f_{\theta}(z)=\lim _{\zeta \rightarrow e^{i \theta}} f(z, \zeta)
$$

exists uniformly in every circle $|z| \leqslant r<1$, and so is a regular function in $|z|<1$. Moreover, for almost every $\theta$ the function $f_{\theta}(z)$ satisfies the condition

$$
\int_{0}^{2 \pi} \log +\left|f_{\theta}\left(r \epsilon^{i x}\right)\right| d w<M<+\infty
$$

Hence, in particular, for every such $\theta$ the limit

$$
f_{\theta x}=\lim _{z \rightarrow e^{i x}} f_{\theta}(z)
$$

exists almost everywhere in $x$.

The point of Theorem $2^{\prime}$ is that under condition (4) the function $f(z, \zeta)$ has an iterat $d$ limit almost everywhere. The extension of Theorem $2^{\prime}$ to general $k \geqslant 2$ is as follows.

4) See Jessen, Marcinkiewicz and Zygmund [1], or Saks [1], p. 147.

5) See Hardy and Littlewood [1], or Zygmund [1], p. 241 sqq. The latter book will henceforth be quoted $T S$.

Fundamenta Mathematicae. T. XxxvI. 
Theorem 2. Let $f\left(z_{1}, z_{2}, \ldots, z_{k}\right)$, regular in $\Gamma_{k}$, satisfy the condition

$$
\int_{0}^{2 \pi} \ldots \int_{0}^{2 \pi} \log +\left|f\left(r_{1} e^{i x_{1}}, \ldots, r_{k} e^{i x_{k}}\right)\right| d x_{1} \ldots d x_{k}<M<\infty \quad\left(r_{1}, \ldots, r_{k}<1\right)
$$

Then for almost every $x_{1}$ the function

$$
f_{x_{1}}\left(z_{2}, \ldots, z_{k}\right)=\lim _{z_{1} \rightarrow e^{i x_{1}}} f\left(z_{1}, z_{2}, \ldots, z_{k}\right)
$$

exists uniformly in every $(k-1)$-cylind $3 r\left|z_{2}\right| \leqslant r_{2}<1, \ldots,\left|z_{k}\right| \leqslant r_{k}<1$, and so is a regutar function of $z_{2}, \ldots, z_{k}$ for $\left|z_{2}\right|<1, \ldots,\left|z_{k}\right|<1$. Moreover, for almost every $x_{1}$,

$$
\int_{0}^{2 \pi} \ldots \int_{0}^{2 \pi} \log +\left|f_{x_{1}}\left(r_{2} e^{i x_{2}}, \ldots, r_{k}, i x_{k}\right)\right| d x_{2} \ldots d x_{k}<M_{x_{1}}<+\infty
$$

It follows from Theorem 2 that

$$
f_{x_{1} x_{3}}\left(z_{3}, \ldots, z_{k}\right)=\lim _{z_{2} \rightarrow e^{i x_{2}}} f_{x_{1}}\left(z_{2}, \ldots, z_{k}\right)
$$

exists almost everywhere in $x_{1}, x_{2}$ and is a regular function of $z_{3}, \ldots, z_{k}$. This argument can be repeated and, in particular, we see that the function $f_{x_{1} x_{2} \ldots x_{k}}$ exists almost everywhere in $Q_{k}$.

Let $\alpha$ be any positive nunber. A fanction $f\left(z_{1}, \ldots, z_{k}\right)$ regular in $\Gamma_{k}$ will be said to belong to the class $H^{\alpha}$, if

$$
\int_{0}^{2 \pi} \ldots \int_{0}^{2 \pi}\left|f\left(r_{1} e^{i x_{1}}, \ldots, r_{k} e^{l x_{k}}\right)\right|^{\alpha} d x_{1} \ldots d x_{k}<M^{\alpha}<+\infty \quad\left(r_{1}, \ldots, r_{k}<1\right) .
$$

Obviously, if $f$ satisfies (8), then the integral (3) is bounded so that the limit $f\left(\epsilon^{i x_{1}}, \ldots, e^{i x_{k}}\right)$ exists almost everywhere.

Theorem 3. If $f\left(z_{1}, \ldots, z_{k}\right)$ is of the class $H^{\alpha}$, then

$$
\lim _{r_{1}, \ldots, r_{k} \rightarrow 1} \int_{0}^{2 \pi} \ldots \int_{0}^{2 \pi}\left|f\left(r_{1} e^{i x_{1}}, \ldots, r_{k} e^{t x_{k}}\right)-f\left(e^{i x_{1}}, \ldots, e^{i x_{k}}\right)\right|^{\alpha} d x_{1} \ldots d x_{k}=0 .
$$

This theorem is a corollary of another one which we shall state in a moment.

If $A_{1}, A_{2}, \ldots, A_{k}$ are sets of points situated respectively in the planes of the variables $z_{1}, z_{2}, \ldots, z_{k}$, then by $A_{1} \times A_{2} \times \ldots \times A_{k}$ we shall mean the set of the systems $\left(z_{1}, z_{2}, \ldots, z_{k}\right)$ such that $z_{1} \in A_{1}$, $z_{2} \in A_{2}, \ldots, z_{k} \in A_{k}$.
Let $0<\eta<R, 0 \leqslant \theta \leqslant 2 \pi$. In what follows, by $\Delta(\theta, \eta, R)$ we shall mean the open domain limited by the tangents from the point $R t^{i \theta}$ to the circle $|\zeta|=\eta$, and by the longer are of that circle between the points of contact. Instead of $\Delta(\theta, \eta, 1)$ we shall often write $\Delta(\theta, \eta)$.

Theorem 4. S'uppose that $f\left(z_{1}, \ldots, z_{k}\right)$ is regular in $\Gamma_{k}$ and satisfies condition (S). Let

$$
\begin{gathered}
F^{\eta}\left(x_{1}, \ldots, x_{k}\right)=\operatorname{Sup}\left|f\left(z_{1}, \ldots, z_{k}\right)\right| \\
\text { for } \quad\left(z_{1}, \ldots, z_{k}\right) \in \Delta\left(x_{1}, \eta\right) \times \ldots \times \Delta\left(x_{k}, \eta\right) .
\end{gathered}
$$

Then

$$
\int_{0}^{2 \pi} \ldots \int_{0}^{2 \pi}\left\{F^{\eta_{1}}\left(x_{1}, \ldots, x_{k}\right)\right\}^{\alpha} d x_{1} \ldots d x_{k} \leqslant\left(A_{\eta, \alpha} M\right)^{\alpha}
$$

with $A_{\eta, c}$ finite and depending on $\eta, \alpha$ only.

That this theorem implies Theorem 3 is immediate since, on account of (11), and of Theorem 1, the integrand in (10) tends to 0 for almost every $\left(x_{1}, \ldots, x_{k}\right)$ and is majorized by an integrable function.

Before we pass to the proofs of the above (and some other) theorems, a few remarks may be useful.

(i) In the case of regular functions $f(\zeta)$ of a single variable, both the theorem of Fatou and its Nevanlinna-Ostrowsk generalization (the latter in a suitable form) can be immediately extended to, say, simply connected domains. It is enough to map conformally the domain considered onto the unit circle, and to "transplant" the function from the former into the latter. By considering subdomains of the given domain we can also considerably relax the condition of simple connectedness. For obvious reasons such an argument is applicable to functions of more than one complex variable in the case of some special domains only. For more general domains in the complex space of 7 dimensions, if we wanted to obtain extensions of the theorems stated above, we should have to give special proofs, imitating to a certain degree the proofs of these theorems ${ }^{6}$ ). This we shall not do here and we concentrate

6) For the behavior of analytic functions in such domains see various papers of Bergman, in particular Bergman [1]. See also Bergman and Marcinkiewicz [1], and Bers [1]. The latter paper contains an extensive list of Bergman's papers in this field. 
on the domains $\Gamma_{k}$ which are the most important ones for the study of power series and of trigonometric series of several variables.

(ii) In the proof of Theorem 1 we shall use the fact that $\log ^{+}\left|f\left(z_{1}, \ldots, z_{k}\right)\right|$ is a subharmonic function of each $z_{j}$, the other $z^{\prime} \mathrm{s}$ beirg fixed. Considerable part of the argument can be immediately extended to multiply subharmonic functions, that is functions of several complex variables, subharmonic in each of these variables. However, here again we shall not go beyond what is strictly needed for the proofs of the theorems enounced, leaving generalizations. aside.

(iii) The case of regular functions $f(\zeta),|\zeta|<1$, of a single complex. variable is exceptional insofar as we have here at our disposal a decomposition of $f$ into a non-vanishing regular function and the Blaschke product formed with the zeros of $f$. If we had $a_{\text {similar }}$ result for funetions of several complex variables, the proofs in that. case would merely be repetitions of proofs for one variable. However, no such decomposition exists for functions of several complex variables, and it is this fact which makes the proof more difficult there (see also Bergman [2], and the remarks in Bochner [1]). Roughly speaking, the proof of Theorem 1 will proceed as follows. First of all, using the fact that $\log +|f|$ is multiply subharmonic we prove Theorem 1 in a weaker form, where the convergence of $f$ along nontangential paths is replaced by the boundedness of $f$ along such paths. At this stage, the usefulness of the mere subharmonic character of $\log ^{+}|f|$ will have been exhausted because for subharmonic functions the most we could hope for would be the existence of limits along radii or slightly more general curves, but not inside angles. (A good illustration here is provided by a result of Littlewood [1]. He proved that if $\omega(\zeta)$ is subharmonic in $|\zeta|<1$, and

if $\int_{0}^{2 \pi} \omega^{+}\left(\varrho \epsilon^{\theta \theta}\right) d \theta=0(1)$ for $\varrho<1$, then the radial limit of $\omega(\zeta)$ exists. almost everywhere. Since $\omega(\zeta)$ can be equal to $-\infty$ in a set dense in $|\zeta|<1$, his result obviously cannot be extended to non-tangential paths. As one easily sees, the situation is not improved even if one considers bounded subharmonic functions $\omega(\zeta)$ ). The passage from the boundedness of $f$ to the existence of a limit will already take into account some specific properties of regular functions. (iv) Theorems 3 and 4 are known for $\alpha>1$, and in this case the result is valid for multiply harmonic functions, that is continuous functions of several complex variables, harmonic in each variable separately. (See e.g. Sokół-Sokołowski [1], Bergman and Marcinkiewicz [1], Bochner [1]). For $\alpha=1$, Bochner [1] proved that $f\left(z_{1}, \ldots, z_{k}\right)$ converges to a limit in the $L$-metric (he did not prove the pointwise convergence). The remaining parts of Theorems 3 and 4 seem to be new.

(v) The plan of the paper is as follows. In Section 2 we give certain lemmas needed for the proofs of our theorems. The proofs of the theorems are completed in Section 3, which also contains some additional remarks about the theorems. Finally, Section 4 contains applications of Theorems 3 and 4 to conjugate functions in the case of several variables. For details we refer the reader to that section.

2. Auxiliary lemmas. Lemma 1. Let $\varphi_{1}(\zeta), \varphi_{2}(\zeta), \ldots$ be functions regular and uniformly bound.d for $|\zeta|<1$. Suppose that the sequence of boundary values $\varphi_{1}\left(\epsilon^{i \theta}\right), \varphi_{2}\left(\iota^{i \theta}\right), \ldots$ converges for every $\theta$ $b \in$ longing to a set $E$ of positive measure $\left.{ }^{7}\right)$. Then for almost every $\theta_{0} \in E$ $\left\{\varphi_{n}(\zeta)\right\}$ converges uniformly in $\Delta\left(\theta_{0}, \eta\right)$, no matter what is $\left.\eta<1^{8}\right)$.

Let us first consider an arbitrary function $\varphi(\zeta)$ regular and satisfying $|\varphi(\zeta)|<1$ for $|\zeta|<1$. Let us suppose that $\left|\varphi\left(\epsilon^{\imath \theta}\right)\right|<\varepsilon$ for $\theta \in E$. The function $\psi(\zeta)=\log |\varphi(\zeta)|$ is a negative subharmonic function in $|\zeta|<1$. Let $P(\varrho, \theta)$ be the Poisson kernel,

$$
P(\varrho, \theta)=\frac{1}{2} \frac{1-\varrho^{2}}{1-2 \varrho \cos \theta+\varrho^{2}} .
$$

7) Each $\varphi_{n}$ obviously has boundary values $\varphi_{n}\left(e^{i \theta}\right)$ almost everywhere. We recall that boundary values are defined as limits along nontangential paths.

8) As I learnt recently, Lemma $I$ is not entirely new. Cotlar and Vignaux $[1]$ had shown that if the assumptions of Lemma 1 are satisfied, and if in addition
the functions $\varphi_{n}$ are ale continuous on an arc of the unit circle containing $E$, then the sequence $\left\{\varphi_{n}(\zeta)\right\}$ converges uniformly over almost every radius terminating in $E$; more precisely, over every radius terminating at a point of density of $E$.

In Lemma 1, the uniform boundedness of the functions $\varphi_{n}(5)$ can be replaced by the condition that the subharmonic functions $\log ^{+}\left|\varphi_{n}(\zeta)\right|$ have a common harmonic majorant $h(\varrho, \theta)$ which is the Poisson integral of an L-integrable function (or, what is the same thing, that the integrals $\int h(\varrho, \theta) d \theta$ are uniformly absolutely continuous). The proof undergoes but little change. 
One easily sees that for $\varrho<1$ one has ${ }^{9}$ )

(12)

$$
\begin{gathered}
\psi\left(\varrho \epsilon^{i \theta}\right) \leqslant \frac{1}{\pi} \int_{0}^{2 \pi} \psi\left(\epsilon^{i u}\right) P(\varrho, \theta-u) d u \\
\leqslant \frac{1}{\pi} \int_{E} \psi\left(\epsilon^{i a}\right) P(\varrho, \theta-u) d u \leqslant-(\log 1 / \varepsilon) \cdot \frac{1}{\pi} \int_{E} P(\varrho, \theta-u) d u .
\end{gathered}
$$

The last integral here is the Poisson integral of the characteristic function $h(\theta)$ of the set $E$. In virtue of the classical result of Fatou, at every point $\theta_{0}$ at which the integral $H(\theta)$ of $h(\theta)$ has a derivative, the Poisson integral of $h$ tends to $H^{\prime}\left(\theta_{0}\right)$ as $\varrho^{i \theta}$ approaches $t^{i \theta_{*}}$. within any $\Delta\left(\theta_{0}, \eta\right)$. In particular, if $\theta_{0}$ is a point of density of $E$, then $H^{\prime}\left(\theta_{0}\right)=1$, and the Poisson integral tends to limit 1 there. Since this integral represents a positive function inside the unit circle, one immediately sees that there is a positive constant $K=K\left(\theta_{0}, E, \eta\right)$ such that

$$
\frac{1}{\pi} \int_{E} P(\varrho, \theta-u) d u \geqslant K \text { for } \zeta=\varrho \epsilon^{i \theta} \in \Delta\left(\theta_{0}, \eta\right) \text {. }
$$

This and (12) imply that

$$
\log |\varphi(\zeta)| \leqslant-K \log (1 / \varepsilon) \text { for } \zeta \in \Delta\left(\theta_{0}, \eta\right) .
$$

The point of this inequality is that if $|\varphi(\zeta)|<1$ for $|\zeta|<1$, and if $\left|\varphi\left(e^{i \theta}\right)\right|$ is uniformly small on $E$, then $|\varphi(\zeta)|$ is small in $\Delta\left(\theta_{0}, \eta\right)$.

It is now easy to complete the proof of Lemma 1. We may suppose that $\left|\varphi_{n}(\zeta)\right|<\frac{1}{2}$ for $|\zeta|<1$ and for all $n$. Hence the functions $\Phi_{m n}(\zeta)=\varphi_{m}(\zeta)-\varphi_{n}(\zeta)$ are absolutely less than 1 in $|\zeta|<1$. Without loss of generality, usirg Egoroff's theorem, we may assume that $\left\{\varphi_{n}\left(\epsilon^{i \theta}\right)\right\}$ converges uniformly on $E$. Then $\left|\Phi_{m n}\left(\epsilon^{i \theta}\right)\right|$ is uniformly small on $E$ for $m$ and $n$ sufficiently large and so, by what has just been proved, $\left|\Phi_{m n}(\zeta)\right|$ is uniformly small in $\Delta\left(\theta_{0}, \eta\right)$ for such $m, \dot{n}$, if $\theta_{0}$ is a point cf density of $E$. This completes the proof of Lemma 1 .

9) The first inequality that follows is obtained from the inequality

$$
\psi\left(\varrho e^{i \theta}\right) \leqslant \frac{1}{2 \pi} \int_{0}^{2 \pi} \psi\left(R e^{i u}\right) \frac{R^{2}-\rho^{2}}{R^{2}-2 R \varrho \cos (\theta-u)+\varrho^{2}} d u . \quad(\rho<R<1)
$$

which is obvious if $\varphi$ has no zeros on $|\xi|=R$. It is enough to make here $R \rightarrow 1$, and to use the very well known Fatou lemma.
The fact that under the conditions of Lemma 1 the sequence $\left\{\varphi_{n}(\zeta)\right\}$ converges urifornly in every circle $|\zeta| \leqslant r_{1}<1$ is, of course, well known. For our pur pose however, it is important to have uniform convergence in the remainirg part of $\Delta\left(\theta_{0}, \eta\right)$.

Remark. As the above proof shows, the Lemma holds if the subscript $n$ terds to a limit, infinite or finite, in a continuos $x a y$. In our applicatiors, $n$ will be a corplex variable, or even a set of conplex variables, ter dir $g$ to a firite limit.

Let $D$ be a domain in the $z$-plare limited by a simple closed curve $C$. A subdomain $D^{\prime}$ of $D$ limited by a simple closed curve $C^{r}$ will be called of type $\Delta$, if

a) $C^{\prime}$ has exactly ore point $z_{0}$ in common with $C$, and otherwise is situated totally in the interior of $C$;

b) the curve $C$ has a targent $t$ at $z_{0}$;

c) the curve $C^{\prime}$ is non-tar gential to $C$ at $z_{0}$.

Condition c) mears that in the neighborhood of $z_{0}$ the curve $C^{r}$ is contained in an argle with vertex at $z_{0}$, situated on one side of $t$, with neither side of the angle on $t$.

Lemma 2. Let $f_{1}(z), f_{2}(z), \ldots$ be a sequence of functions rcgular and uniformly bound $\bar{d}$ in a domain $D$ limited by a simple closed and rectifiable curve $C$. Suppose that the boundary values of the $f_{n}(z)$ exists at every point of a set $E$. situatid on $C$ and of positive linear measure, and that these boundary values converge to a limit, as $n \rightarrow \infty$, at every point of $E$. Then almost every point $z_{0}$ of $E$ has the following property: the sequence $\left\{f_{n}(z)\right\}$ converges uniformly inside every suldomain of $D$ having $z_{0}$ on its boundary and of type $\Delta$.

This lemma can be easily deduced from Lemma 1. For let $z=\mu(\zeta)$ be a function regular in the circle $|\zeta|<1$ and mapping that circle conformally on to $D$. The functiors $\varphi_{n}(\zeta)=f_{n}[\mu(\zeta)]$ are regular and uniformly bounded in $|\zeta|<1$. Since the mappir $g$ can be extended in a continuous ard ore-one way to the closed circle $|\zeta| \leqslant 1$, and is conformal with respect to non-tar gertial paths at alnost every point of $|\zeta|=1$, and sirce the sets of linear neasure zero on either boundary are mapped into sets of measure zero, one sees immediately that there is a set $E^{\prime}$ of positive measure situated on $|\zeta|=1$ and such that at almost every point of it the boundary values of the functions $\varphi_{n}(\zeta)$ exist and converge to a limit as $n \rightarrow \infty$. The set $E^{\prime}$ is merely the image of $E$ through the mappirg $z=\mu(\zeta)$. If we omit from $E^{\prime}$ a subset of measure zero, the $\varphi_{n}$ will possess the required 
properties at every point of $E^{\prime}$. Hence, by Lemma 1, for almost every point $\epsilon^{i \theta_{0}} \epsilon E^{\prime}$ the sequence $\left\{\varphi_{n}(\zeta)\right\}$ converges uniformly in every $\Delta\left(\theta_{0}, \eta\right)$. If the mapping is corformal at $\zeta_{0}=e^{i \theta_{0}}$, this implies the uniform convergence of $\left\{f_{n}(z)\right\}$ in every subdomain $D^{\prime}$ of $D$ of type $\Delta$ and having $z_{0}=\mu\left(c^{i \theta_{0}}\right)$ on its boundary. Obviously almost every point $z_{0} \in E$ has the required property, and the lemma follows.

Also this lemma holds if $n$ is a continuous variable (or a set of variables) tending to a limit.

Lemma 3. Let $U_{\boldsymbol{Z}}(\zeta)$ be a family of subharmonic functions in $|\zeta|<1$ dependirg on a real-valued parameter $\lambda$ and non-decreasing in $\lambda$ for every fixed $\zeta,|\zeta|<1$. If

$$
\int_{0}^{2 \pi} J_{2}\left(\varrho \epsilon^{i \theta}\right) d \theta<M<+\infty \quad(0 \leqslant \varrho<1)
$$

with $M$ independent of $\lambda$ and $\varrho$, then there is a harmonic function $H(\zeta)$ in $|\zeta|<1$ majorizing all the $U_{2}(\zeta)$ there.

This is a classical result for harmonic functions. The passage to subharmonic functions is immediate.

Lemma 4. Under the assumptions of Theorem 1, for almost every point $\left(x_{1}^{0}, x_{2}^{0}, \ldots, a_{k}^{0}\right)$, the function $f\left(z_{1}, z_{2}, \ldots, z_{k}\right)$ is bound.d in

$$
\left.\Delta\left(x_{1}^{0}, \eta\right) \times \Delta\left(x_{2}^{0}, \eta\right) \times \ldots \times \Delta\left(x_{k}^{0}, \eta\right), \quad \text { whatever } \eta<1^{11}\right) .
$$

Let $\eta$ and $\lambda$ denote two positive numbers less than 1 . If $u(z)$ is a non-negative harmonic function in $|z|<\lambda$, and continuous for $|z| \leqslant \lambda$ we set

$$
\nabla^{\eta, \lambda}(x)=\operatorname{Max} u(z) \quad \text { for } z \in \Delta(x, \lambda \eta, \lambda) .
$$

We shall need two inequalities for $\left.U^{\eta 2, \lambda}(x)^{10}\right)$. The first is

$$
\left\{\int_{0}^{2 \pi}\left[U^{\eta \lambda, \lambda}(x)\right]^{\delta} d x\right\}^{1 / \delta} \leqslant B_{\eta, \delta} \int_{0}^{2 \pi} u\left(\lambda \epsilon^{i x}\right) d x,
$$

10) Both inequalities easily follow from the principal results of $\mathrm{Hardy}$ and Littlewood [I], though neither is stated there explicitly. A proof of (15) will be found in TS, p. 248 .

11) This lemma, whose refinement to Theorem 1 represents the main result of the present paper, was presented at the meeting of the Mathematical Club of the University of Michigan March, 1942. where $\delta$ is any positive number less than 1 . The second inequality is

$$
\int_{0}^{2 \pi} \chi_{\alpha-1}\left\{U^{\eta \lambda, 2}(x)\right\} d x \leqslant A_{\eta, \alpha} \int_{0}^{2 \pi} \chi_{\alpha}\left\{u\left(\lambda \epsilon^{i x}\right)\right\} d x+A_{\eta, \alpha}
$$

where $\alpha \geqslant 1$ and

$$
\chi_{\alpha}(t)=t \log ^{\alpha}(t+1),
$$

Here and in what follows by letters $A, B, \ldots$ with various subscripts we shall denote constants, not necessarily always the same, depending only on the parameters exhibited in the subscripts.

It is seen immediately that the inequalities (15) and (16) remain valid if $u(z)$ is a function non-negative, continuous and subharmonic in $|z|<1$. The condition of continuity can be dropped here, but the point is without importance for us, and the passage from subharmonic functions to their harmonic majorants is particularly simple if the former are continuous. For it is enough to apply the inequalities (15) and (16) to the function harmonic in $|z|<\lambda$, continuous in $|z| \leqslant \lambda$ and coinciding with the given subharmonic function 26 on $|z|=\lambda$, and then to use the fact that this harmonic function majorizes $u$ in $|z|<\lambda$.

$$
\text { Let us write }
$$

$$
\log +\left|f\left(z_{1}, \ldots, z_{k}\right)\right|=u\left(z_{1}, \ldots, z_{k}\right)
$$

Taking, as before, $0<\eta<1,0<\lambda<1$, we set

$$
\begin{aligned}
& \nabla_{x_{1}}^{\eta \lambda, \lambda}\left(z_{2}, \ldots, z_{k}\right)=\operatorname{Max} u\left(z_{1}, \ldots, z_{k}\right) \quad \text { for } z_{1} \in \Delta\left(x_{1}, \eta \lambda, \lambda\right) \text {, } \\
& D_{x_{1} x_{2}}^{\eta \lambda, \lambda}\left(z_{3}, \ldots, z_{k}\right)=\operatorname{Max} U_{x_{1}}^{\eta \lambda, \lambda}\left(z_{2}, \ldots, z_{k}\right) \quad \text { for } z_{2} \in \Delta\left(x_{2}, \eta \lambda, \lambda\right) \text {, } \\
& \text {. . . . . . . . . . } \\
& D_{x_{1} \ldots x_{k}}^{\eta \lambda, \lambda}=\operatorname{Max} D_{x_{1} \ldots x_{k-1}}^{\eta \lambda, \lambda}\left(z_{k}\right) \quad \text { for } z_{k} \in \Delta\left(x_{k}, \eta \lambda, \lambda\right) \text {. }
\end{aligned}
$$

Each of the expressions on the left is a continuous and subharmonic function of each of the variables $z_{j}$ in parenthesis. If no confusion arises, we shall omit the superseripts $\eta \lambda, \lambda$ in notation. By (15),

$$
\left\{\int_{0}^{2 \pi}\left(U_{x_{1} \ldots x_{k}}\right)^{\delta} d x_{k}\right\}^{1 / \delta} \leqslant B_{\eta, \delta} \int_{0}^{2 \pi} U_{x_{1}, \ldots x_{k-1}}\left(\lambda e^{i x_{k}}\right) d x_{k} .
$$

If we integrate both sides with respect to $x_{1}, \ldots, x_{k-1}$ and apply Hölder's inequality to the left side, we get

(18 a) $\left\{\int_{0}^{2 \pi} \ldots \int_{0}^{2 \pi}\left(U_{x_{1} \ldots x_{k}}\right)^{\delta} d x_{1} \ldots d x_{k}\right\}^{1 / \delta} \leqslant B_{\eta, \delta} \int_{0}^{2 \pi} \ldots \int_{0}^{2 \pi} U_{x_{1} \ldots x_{k-1}}\left(\lambda \epsilon^{i x_{k}}\right) d x_{1} \ldots d x_{k}$. 
Similarly, by (16),

$\int_{0}^{2 \pi} U_{x_{1} \ldots x_{k-1}}\left(\lambda e^{i x_{k}}\right) d x_{k-1} \leqslant A_{\eta, 1} \cdot \int_{0}^{2 \pi} x_{1}\left\{U_{x_{1} \ldots x_{k-2}}\left(\lambda e^{i x_{k-1}}, \lambda e^{i x_{k}}\right)\right\} d x_{k-1}+A_{\eta_{1}, 1}$, and integration with respect to $x_{1}, \ldots, x_{k-2}, x_{k}$ gives

$\int_{0}^{2 \pi} \ldots \int_{0}^{2 \pi} U_{x_{1} \ldots x_{k-1}}\left(\lambda e^{i x_{k}}\right) d x_{1} \ldots d x_{k} \leqslant$

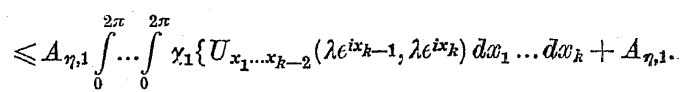

Proceeding in the same way and constantly using (16) we get

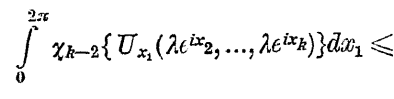

$$
\leqslant A_{\eta, k-1} \int_{0}^{2 \pi} \chi_{k-1}\left\{u\left(\lambda e^{\left.i x_{1}, \ldots, \lambda t^{i x_{k}}\right)}\right\} d x_{1}+A_{\eta, k-1},\right.
$$

and so

(19)

$$
\begin{aligned}
& \int_{0}^{2 \pi} \ldots \int_{0}^{2 \pi} \chi_{k-2}\left\{U_{x_{1}}\left(\lambda e^{i x_{2}}, \ldots, \lambda e^{i x_{k}}\right)\right\} d x_{1} \ldots d x_{k} \leqslant \\
& \leqslant A_{\eta, k-1} \int_{0}^{2 \pi} \ldots \int_{0}^{2 \pi} \chi_{k-1}\left\{u\left(\lambda e^{i x_{1}}, \ldots, \lambda e^{i x_{k}}\right)\right\} d x_{1} \ldots d x_{k}+A_{\eta, k-1} .
\end{aligned}
$$

Using this chain of inequalities from (18a) through (19) we find

(20)

$$
\begin{aligned}
& \left\{\int_{0}^{2 \pi} \ldots \int_{0}^{2 \pi}\left(U_{x_{1} \ldots x_{k}}^{2 \eta, \lambda}\right)^{\delta} d x_{1} \ldots d x_{k}\right\}^{1 / \delta} \leqslant \\
& \leqslant C_{\eta, \delta, k} \int_{0}^{2 \pi} \ldots \int_{0}^{2 \pi} x_{k-1}\left\{u\left(\lambda e^{i x_{1}}, \ldots, \lambda \epsilon^{i x_{k}}\right)\right\} d x_{1} \ldots d x_{k}+C_{\eta, \delta, k} .
\end{aligned}
$$

Let us now observe that the boundedness of the integral (3) is equivalent to an inequality

(21) $\int_{0}^{2 \pi} \ldots \int_{0}^{2 \pi} x_{k-1}\left\{u\left(r_{1} \epsilon^{i x_{1}}, \ldots, r_{k} \epsilon^{i x_{k}}\right)\right\} d x_{1} \ldots d x_{k}<M<+\infty \quad\left(r_{1}, \ldots, r_{k}<1\right)$

Hence the left side of (20) never exceeds $C_{\eta, \delta, h} M+C_{\eta, \delta, k \text { a }}$ The integrand on the left is a non-decreasing function of $\lambda$. Hence, making $\lambda$ tend to 1 and setting

$$
D_{x_{1} \ldots x_{k}}^{\eta}=\lim _{\lambda \rightarrow 1} U_{x_{1} \ldots x_{k}}^{2 \eta, \lambda}
$$

we get

$$
\left\{\int_{0}^{2 \pi} \ldots \int_{0}^{2 \pi}\left(U_{x_{1} \ldots x_{k}}^{\eta}\right)^{\delta} d x_{1} \ldots d x_{k}\right\}^{1 / \delta} \leqslant C_{4 ; \delta, k} M+C_{\eta, \delta, k}
$$

Hence, in particular,

$$
U_{x_{1} \ldots x_{k}}^{\eta}<+\infty
$$

for almost every point $x_{1}, \ldots, x_{k}$. Taking for $\eta$ a sequence of values tending to 1 , we obtain Lemma 4 since, as immediately seen, $U_{x_{1} \ldots x_{k}}$ is the upper bound of $u=\log ^{+}|f|$ in the domain (14)

Incidentally, we also proved that under the assumptions of Theorem 1 the function $U_{x_{1} \ldots x_{k}}$ belongs to $L^{\delta}$ for every $\delta<1$.

3. Proofs of Theorems 1,2 and 4. We begin with the proof of Theorem 2. Let $u(z)$ be subharmonic, non-negative and, say, continuous for $|z|<1$. Let $0<r_{0}<r<1$. Then

$$
2 \pi u(z) \leqslant \frac{r+r_{0}}{r-r_{0}} \int_{0}^{2 \pi} u\left(r \epsilon^{i x}\right) d x \quad \text { for }|z| \leqslant r_{0^{*}}
$$

For $u$ harmonic, this is a classical inequality. The extension to subharmonic functions is immediate.

For fixed values of $z_{2}, \ldots, z_{k}$, each absolutely less than 1 , the function $\log +\left|f\left(z_{1}, \ldots, z_{k}\right)\right|$ is subharmonic in $z_{1}$. Hence

$$
I_{r}\left(z_{1}\right)=\int_{0}^{2 \pi} \ldots \int_{0}^{2 \pi} \log +\left|f\left(z_{1}, r \epsilon^{i x_{2}}, \ldots, r \epsilon^{i x_{k}}\right)\right| d x_{2} \ldots d x_{k}
$$

is a function of $z_{1}$ subharmonic and continuous in $\left|z_{1}\right|<1$, for every value of the parameter $r, 0 \leqslant r<1$. It is a non-decreasing function of $r$. To prove the latter, it is enough to show that if we replace in the integral the numbers $r_{t} t_{2}, \ldots, r_{t} x_{k}$ by $r_{2} t^{i x_{2}}, \ldots, r_{k} t^{i x_{k}}$ the resulting expression is a non-decreasing function of $r_{2}, \ldots, r_{k}$. This is seen, for example in the case $r_{2}$, from the fact that $\int_{0}^{2 \pi} \log +\left|f\left(z_{1}, r_{2} e^{i x_{2}}, \ldots, r_{k} \epsilon^{i x_{k}}\right)\right| d x_{2}$ is a non-decreasing function of $r_{2}$ for every system of $z_{1}, x_{3}, \ldots, x_{k}$ and so remains a non-decreasing function of $r_{2}$ after integration with respect to $x_{3}, \ldots, x_{k}$ over the interval $(0,2 \pi)$. 
The inequality (7) gives

$$
\int_{0}^{2 \pi} I_{r}\left(r_{1} \epsilon^{i x_{1}}\right) d x_{1}<M<+\infty
$$

so that, by Lemma 3, there is a harmonic and non-negative function $H\left(z_{1}\right)$ majorizing all the $I_{r}\left(z_{1}\right)$ in $\left|z_{1}\right|<1$. The function $H\left(z_{1}\right)$ has a non-tangential limit almost everywhere. In particular, for almost every $x_{1}$ there is a finite constant $M\left(x_{1}, \eta\right)$ such that $H\left(z_{1}\right)<M\left(x_{1}, \eta\right)$ for $z_{1} \in \Delta\left(x_{1}, \eta\right), \eta<1$. Hence

$$
I_{r}\left(z_{1}\right)=\int_{0}^{2 \pi} \ldots \int_{0}^{2 \pi} \log ^{+}\left|f\left(z_{1}, r \epsilon^{i x_{2}}, \ldots, r \epsilon^{i x_{k}}\right)\right| d x_{2} \ldots d x_{k}<M\left(x_{1}, \eta\right)
$$

for $z_{1} \in \Delta\left(x_{1}, \eta\right)$.

Since, for $z_{1}$ fixed, $\log ^{+}\left|f\left(z_{1}, z_{2}, \ldots, z_{k}\right)\right|$ is a subharmonic function of each of the variables $z_{2}, \ldots, z_{k}$, repeated application of (23) gives the inequalities

$$
\begin{aligned}
& \log ^{+}\left|f\left(z_{1}, z_{2}, \ldots, z_{k}\right) \leqslant \frac{1}{2 \pi} \frac{r+r_{0}}{r-r_{0}} \int_{0}^{2 \pi} \log +\right| f\left(z_{1}, r \epsilon^{i x_{2}, z_{3}}, \ldots, z_{k}\right) \mid d x_{2}
\end{aligned}
$$

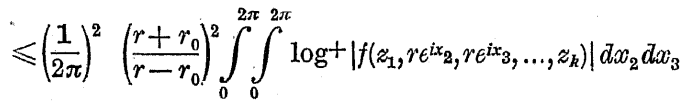

$$
\begin{aligned}
& \text { •. . . . . . . . . . . . . . . . . . } \\
& \leqslant\left(\frac{1}{2 \pi}\right)^{k-1}\left(\frac{r+r_{0}}{r-r_{0}}\right)^{k-1} \int_{0}^{2 \pi} \ldots \int_{0}^{2 \pi} \log +\left|f\left(z_{1}, r \epsilon^{i x_{2}}, \ldots, r \epsilon^{i x_{k}}\right)\right| d x_{2} \ldots d x_{k} \\
& =\left(\frac{1}{2 \pi}\right)^{k-1}\left(\frac{r+r_{0}}{r-r_{0}}\right)^{k-1} I_{r}\left(\tilde{z}_{1}\right), \text { if }\left|z_{2}\right| \leqslant r_{0}, \ldots,\left|z_{k}\right| \leqslant r_{0}, 0<r_{0}<r<1 .
\end{aligned}
$$

Let us fix a value $x_{1}^{0}$ of $x_{1}$ for which we have (24). Making $r$ tend to 1 we get

$$
\begin{aligned}
& \log \left|f\left(z_{1}, z_{2}, \ldots, z_{k}\right)\right| \leqslant\left(\frac{1}{2 \pi}\right)^{k-1}\left(\frac{1+r_{0}}{1-r_{0}}\right)^{k-1} M\left(x_{1}^{0}, \eta\right), \\
& \quad \text { for } z_{1} \in \Delta\left(x_{1}^{0}, \eta\right),\left|z_{2}\right| \leqslant r_{0}, \ldots,\left|z_{k}\right| \leqslant r_{0} .
\end{aligned}
$$

Hence $f\left(z_{1}, z_{2}, \ldots, z_{k}\right)$ is bounded for $\left|z_{2}\right|, \ldots,\left|z_{k}\right|$ not exceeding $r_{0}$ and for $z_{1} \in \Delta\left(x_{1}^{0}, \eta\right)$, whatever $r_{0}<1$ and $\eta<1$. It follows that $f\left(z_{1}, z_{2}, \ldots, z_{k}\right)$ is equicontinuous in the variables $z_{2}, \ldots, z_{k}$ satisfying $\left|z_{2}\right| \leqslant r_{0}, \ldots,\left|z_{k}\right| \leqslant r_{0}$, provided the parameter $z_{1}$ remains in $\Delta\left(x_{1}^{0}, \eta\right)$.
We shall now prove the existence of $f_{x_{1}}\left(z_{2}, \ldots, z_{k}\right)$ for almost every $x_{1}$ and uniformly in $\left|z_{2}\right| \leqslant r_{0}, \ldots,\left|z_{k}\right| \leqslant r_{0}$. For this purpose, on account of the equicontinuity just established, it is enough to show that there is a sequence of points $\left(\hat{k}_{2}^{j}, \ldots, z_{k}^{j}\right), j=1,2, \ldots$, dense in the $(k-1)$-cylinder

$$
\left|z_{2}\right|<1, \ldots,\left|z_{k}\right|<1
$$

and such that, for every $j$, and almost every $x_{1}$ the function $f\left(z_{1}, z_{2}^{j}, \ldots, z_{k}^{j}\right)$ tends to a limit as $z_{1}$ tends to $\epsilon^{i x_{1}}$ within $\Delta\left(x_{1}, \eta\right)$. The set $E_{j}$ of the values $x_{1}$ for which this takes place and which is of measure $2 \pi$, depends on $j$, but the set $E=E_{1} E_{2} \ldots$ is still of measure $2 \pi$; and in $E$ the limit of $f\left(z_{1}, \hat{z}_{2}^{j}, \ldots, z_{k}^{j}\right)$ will exist for every $j$.

It is of course enough to prove that for every fixed set of the radii $r_{2}^{0}, \ldots, r_{k}^{0}$ less than 1 , almost every system of amplitudes $\left(x_{2}^{0}, \ldots, x_{k}^{0}\right)$ has the property that $f\left(z_{1}, r_{2}^{0}, i x_{2}^{0}, \ldots, r_{k}^{0} i x_{k}^{0}\right)$ tends a limit as $z_{1}$ approaches almost every point $\epsilon^{2 x_{1}}$. For this purpose let us write $(7)$ in the form

$$
\int_{0}^{2 \pi} \ldots \int_{0}^{2 \pi}\left\{\int_{0}^{2 \pi} \log ^{+}\left|f\left(r_{1} e^{i x_{1}}, r_{2}^{0} e^{i x_{2}}, \ldots, r_{k}^{0} e^{i x_{k}}\right)\right| d x_{1}\right\} d u_{2} \ldots d x_{k}<M .
$$

The inner integral here is a non-decreasing function of $r_{1}$. It. follows that for almost erery point $x_{2}^{0}, \ldots, x_{k}^{0}$ we have

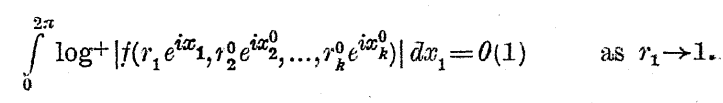

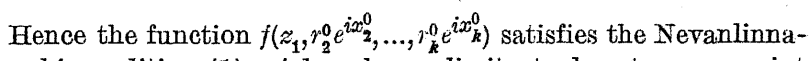
Ostrowski condition (1), and so has a limit at almost every point. of the unit circle. This completes the proof that $f_{x_{1}}\left(z_{2}, \ldots, z_{k}\right)$ exists for almost every $x_{1}$, and uniformily in $\left|z_{2}\right| \leqslant r_{0}, \ldots,\left|z_{k}\right| \leqslant r_{0}$.

We have already used the fact that if in the integral in (24)

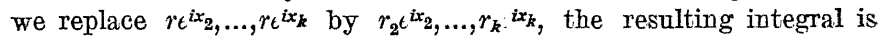
a non-decreasing function of $r_{2}, \ldots, r_{k}$. Hence (24) implies

$\int_{0}^{2 \pi} \ldots \int_{0}^{2 \pi} \log +\left|f\left(z_{1}, r_{2} \epsilon^{i x_{2}}, \ldots, r_{k} \epsilon^{i x_{k}}\right)\right| d x_{2} \ldots d x_{k}<M\left(x_{1}, \eta\right), \quad$ for $z_{1} \in \Delta\left(x_{1}, \eta\right)$ and for every set of numbers $r_{2}, \ldots, r_{k}$ less than 1 . If $x_{1}$ also has the property that $f_{x_{1}}\left(z_{2}, \ldots, z_{k}\right)$ exists in the sense just established, the 
last inequality gives

$$
\int_{0}^{2 \pi} \ldots \int_{0}^{2 \pi} \log +\left|f_{x_{1}}\left(r_{2} \epsilon^{\ell x_{2}}, \ldots, r_{k} \epsilon^{\ell x_{k}}\right)\right| d x_{2} \ldots d x_{k} \leqslant M\left(x_{1}, \eta\right) \quad\left(r_{2}, \ldots, r_{k}<1\right) .
$$

This completes the proof of Theorem 2 .

We now pass to the proof of Theorem 1. The proof will be by induction from $k-1$ to $k$. For $k=1$, Theorem 1 reduces to the NevanIinna-Ostrowski theorem.

Let $\chi(t)$ be a non-negative, increasing and convex function. of $t \geqslant 0$, and suppose that $f\left(z_{1}, \ldots, z_{k}\right)$ is a function regular in the $k$-cylinder $\Gamma_{k}$ and satisfying the condition

$$
\int_{0}^{2 \pi} \ldots \int_{0}^{2 \pi} x\left\{\log ^{+}\left|f\left(r_{1} \epsilon^{i x_{1}}, \ldots, r_{k} \epsilon^{i x_{k}}\right)\right|\right\} d x_{1} \ldots d x_{k}=0(1)
$$

for $r_{1}, \ldots, r_{k}<1$. Since $\chi(t)>$ kt for large $t$, where $z_{6}$ is a positive constant, condition (26) implies an inequality (7). In particular, for almost every $x_{1}$ the function

$$
f_{x_{1}}\left(z_{2}, \ldots, z_{k}\right)=\lim _{z_{i} i x_{1}} f\left(z_{1}, \ldots, z_{k}\right)
$$

exists and is regular for $\left|z_{2}\right|<1, \ldots,\left|z_{k}\right|<1$. Moreover, for almost every $x_{1}$ we shall have not only the inequality $(7 \mathrm{a})$ but the stronger inequality

$$
\int_{0}^{2 \pi} \ldots \int_{0}^{2 \pi} x\left\{\log ^{+} f_{x_{1}}\left(r_{2} e i x_{2}, \ldots, r_{k} \epsilon^{i x_{k}}\right) \mid\right\} d x_{2} \ldots d x_{k}<M_{x_{1}}<\infty .
$$

(In particular, if $f\left(z_{1}, \ldots, z_{k}\right) \epsilon H^{\alpha}$, so does $f_{x_{1}}\left(z_{2}, \ldots, z_{k}\right)$ for almost every $x_{1}$ ).

The proof of this is merely a repetition of the proof of Theorem 2 if we replace in it $\log ^{+}|f|$ by $x\left(\log ^{+}|f|\right.$ ) (the proof is even slightly shorter because the existence of (27) has already been established). For the only property of $\log +|f|$ we used was the subharmonic character with respect to each of the variables $2_{1}, \ldots, z_{k}$, a property which is obviously shared by $\chi\left(\log ^{+}|f|\right)$.

Let us now take for $\chi(t)$ the function $t \log ^{k-1}(t+1)$. Under the assumptions of Theorem 1 condition (26) is then satisfied, and so for almost every $x_{1}$ we have (28), which is equivalent to

$\int_{0}^{2 \pi} \ldots \int_{0}^{2 \pi} \log ^{+}|| f_{x_{1}}\left(r_{2} e^{i x_{2}}, \ldots, r_{k} e^{i x_{k}}\right) \mid$

$$
\left\{\log +\log ^{+}+\left|f_{x_{1}}\left(r_{2} \epsilon^{i x_{2}}, \ldots, r^{k} \epsilon^{i x_{k}}\right)\right|\right\}^{k-1} d x_{2} \ldots d x_{k}<M_{x_{1}}<\infty
$$

(with $M_{x_{1}}$ not necessarily the same as before). In particular, we have $\int_{0}^{2 \pi} \ldots \int_{0}^{2 \pi} \log +\left|f_{x_{k}}\left(r_{2} e^{i x_{2}}, \ldots, r_{k} c^{i x_{k}}\right)\right|$

$$
\left\{\log +\log +\left|f_{x_{1}}\left(r_{2} \epsilon^{t x_{2}}, \ldots, r_{k} \epsilon^{i x_{k}}\right)\right|\right\}^{k-2} d x_{2} \ldots d x_{k}<H_{x_{1}}<\infty
$$

for almost every $x_{1}$. Hence, if we assume the validity of Theorem 1 with $k$ replaced by $k-1$, we get that for almost erery $x_{1}$ the nontangential limit

$$
f_{x_{1}}\left(e^{i x_{2}}, \ldots, e^{i x_{k}}\right)=\lim _{z_{2} \rightarrow e^{i x_{2}, \ldots, z_{k} \rightarrow e^{i x_{k}}}} f_{x_{1}}\left(\tilde{z}_{2}, \ldots, z_{k}\right)
$$

exists almost everywhere in $\left(x_{2}, \ldots, x_{k}\right)$.

Let us now consider any point $\left(x_{2}^{0}, \ldots, x_{k}^{0}\right)$ with the following properties:

(i) For almost every $x_{1}$ the function $f\left(z_{1}, \ldots, z_{k}\right)$ is bounded in

$$
\Delta\left(x_{1}, \eta\right) \times \Delta\left(x_{2}^{0}, \eta\right) \times \ldots \times \Delta\left(x_{k}^{0}, \eta\right)
$$

whaterer $\eta<1$;

(ii) $f_{x_{1}}\left(e^{i x_{2}^{0}}, \ldots, e^{i x_{k}^{0}}\right)$ (see (29)) exists for almost every $x_{1}$.

Almost every point $\left(x_{2}^{0}, \ldots, x_{k}^{0}\right)$ satisfies condition (i), and almost every such point satisfies condition (ii), and so also conditions (i) and (ii) simultaneously. Let us fix such a point $\left(x_{2}^{0}, \ldots, x_{k}^{0}\right)$.

Let us also fix an $\eta, 0<\eta<1$. On account of condition (i),

a) there is a perfect set $P$ of measure arbitrarily close to $2 \pi$ and such that $f\left(z_{1}, \ldots, z_{k}\right)$ is uniformly bounded in (30) for $x_{\overline{1}} \in P$.

We mar also assume that

b) the function $f_{x_{1}}\left(z_{2}, \ldots, z_{k}\right)$ exists for every $x_{\perp} \in P$ and is regular for $\left|z_{2}\right|<1, \ldots,\left|z_{k}\right|<1$;

c) $f_{x_{1}}\left(e^{i x_{2}^{0}}, \ldots, e^{i x_{k}^{0}}\right)$ exists for $x_{1} \in P$.

Let us now set

$$
D=\sum_{x_{1} \in P} \Delta\left(x_{1}, \eta\right)
$$

Thus $D$ is an open domain of a (familiar) starlike shape limited by a simple closed and rectifiable curve $O$. The latter has the points $e^{i x_{1}}, x_{1} \in P$, and only such points, in common with the circumference $\left|z_{1}\right|=1$. The function $f\left(z_{1}, \ldots, z_{k}\right)$ is bounded for $z_{1} \in D$ and $\left(z_{2}, \ldots, z_{k}\right) \in \Delta\left(x_{2}^{0}, \eta\right) \times \ldots \times \Delta\left(x_{k}^{0}, \eta\right)$. Let us consider here $\left(z_{2}, \ldots, z_{k}\right)$ as a parameter tending to $\left(e^{i x_{2}^{0}}, \ldots, e^{i x_{k}^{0}}\right)$. Thus we have a family of functions regular and uniformly bounded for $z_{1} \in D$. On account 
of property b), each function has a limit $f_{x_{1}}\left(z_{2}, \ldots, z_{k}\right)$ at every point $\epsilon^{i x_{1}}$ with $x_{1} \in P$, and on account of $c$ ) these boundary values converge on $P$ for $\left(z_{2}, \ldots, z_{k}\right) \rightarrow\left(e^{i x_{2}^{0}}, \ldots, e^{\left.i x_{k}^{0}\right)}\right.$. On account of Lemma 2 , for almost every $x_{1} \in P$ the function $f\left(z_{1}, z_{2}, \ldots, z_{k}\right)$ tends to a limit as $\left(z_{2}, \ldots, z_{k}\right)$ tends to $\left(e^{\left.i x_{2}^{0}, \ldots, e^{i x_{k}^{0}}\right)}\right.$ and $z_{1}$ approaches $\epsilon^{i x_{1}}$ from within of a subdomain of $D$ of type $\Delta$. For almost every $x_{1} \in P$ the tangents to $\left|z_{1}\right|=1$ and to $C$ coincide (they certainly coincide, though this is not important for us, at every point of density of $P$ ). Thus for almost every point of $P$, and no matter what is $\eta^{\prime}<1$, the function $f\left(z_{1}, \ldots, z_{k}\right)$ tends to a limit as $z_{1}$ tends to $\epsilon^{i x_{1}}$ within $\Delta\left(x_{1}, \eta^{\prime}\right)$ and $\left(z_{2}, \ldots, z_{k}\right)$ tends to $\left(e^{\left.i x_{2}^{0}, \ldots, e^{i x_{k}^{0}}\right)}\right.$ within $\Delta\left(x_{2}^{0}, \eta\right) \times \ldots \times \Delta\left(x_{k}^{0}, \eta\right)$. This property holds for almost every $x_{1}$ since the measure of $P$ is arbitrarily close to $2 \pi$. Takirg now $\eta$ arbitrarily close to 1 we conclude that for almost every $\left(x_{2}^{0}, \ldots, x_{k}^{0}\right)$ we can find a set of measure $2 \pi$ of values $x_{1}^{0}$ such that no matter what is $\eta<1$, the function $f\left(z_{1}, \ldots, z_{k}\right)$ tends to a limit as $\left(z_{1}, \ldots, z_{k}\right)$ tends to $\left(e^{i x_{1}^{0}}, e^{\left.i x_{2}^{0}, \ldots, e^{i x_{k}^{0}}\right)}\right.$ remaining within $\Delta\left(x_{1}^{0}, \eta\right) \times \Delta\left(x_{2}^{0}, \eta\right) \times \ldots \times \Delta\left(x_{k}^{0}, \eta\right)$.

Thus we proved the existence of the limit $f\left(e^{i x_{1}^{0}}, \ldots, e^{i x_{k}^{0}}\right)$ for almost every point $\left(x_{1}^{0}, \ldots, x_{k}^{0}\right)$, under the assumption that Theorem 1 is true with $k$ replaced by $k-1$. Since the assumption is valid for $k=2$, Theorem 1 is established completely.

We now pass to the proof of Theorem 4 . We restrict ourselves to the case $k=2$ which is entirely typical.

Let us consider any function $u(z)$ subharmonic and, say, continuous in $|z|<1$. Usirg the same notation as in the proof of Lemma 4, we have the inequality

$$
\int_{0}^{2 \pi}\left\{U^{\eta, \lambda}(x)\right\}^{p} d x \leqslant A_{\eta}\left(\frac{p}{p-1}\right)^{p} \int_{0}^{2 \pi} u^{p}\left(\lambda \epsilon^{i x}\right) d x \quad(p>1),
$$

due to Hardy and Littlewood (a proof 'can also be found in $T S$, p. 248).

Let now $u(z, \zeta)$ be non-negative, continuous and subharmonic in $z$ and $\zeta$ separately, for $|z|<1,|\zeta|<1$. Let $0<\eta<1,0<\lambda<1$. Let

(31) $U^{\eta 2, \lambda}(x, \theta)=\operatorname{Max} u(z, \zeta)$ for $z \in \Delta(x, \lambda \eta, \lambda), \quad \zeta \in \Delta(\theta, \lambda \eta, \lambda)$.
We shall prove that

(32) $\int_{0}^{2 \pi} \int_{0}^{2 \pi}\left\{U^{\eta \lambda, \lambda}(x, \theta)\right\}^{p} d x d \theta \leqslant\left\{A_{u}\left(\frac{p}{p-1}\right)^{\rho}\right\}^{2} \int_{0}^{2 \pi} \int_{0}^{2 \pi} u u^{p}\left(\lambda t^{i x}, 2 t^{i \theta}\right) d x d \theta(p>1)$.

For, given any $\zeta,|\zeta|<1$, let us set

$$
V \eta^{2, \lambda}(x, \zeta)=\operatorname{Max} u(z ; \zeta) \text { for } z \in \mathcal{A}(x, \eta \lambda, \lambda) \text {. }
$$

Then

$$
U^{n \lambda, \lambda}(x, \theta)=\operatorname{Max} \operatorname{Tr}^{r} \lambda, \lambda(x, \zeta) \text { for } \zeta \in \Delta(\theta, \eta \lambda, \hat{\lambda}) \text {. }
$$

Since, for $\eta, \lambda, x$ fixed, $V r^{2}, 2(x, \zeta)$ is subharmonic and continuous in $|\xi|<1$,

$$
\int_{0}^{2 \pi}\left\{U^{\eta \lambda, \lambda}(x, \theta)\right\}^{p} d \theta \leqslant A_{\eta}\left(\frac{p}{p-1}\right)^{p} \int_{0}^{2 \pi}\left\{F^{\left.\eta \lambda, \lambda\left(x, \lambda^{i \theta}\right)\right\}^{p}} d \theta\right.
$$

or, integratirg both sides with respect to $x$ and inverting the order of integration on the right,

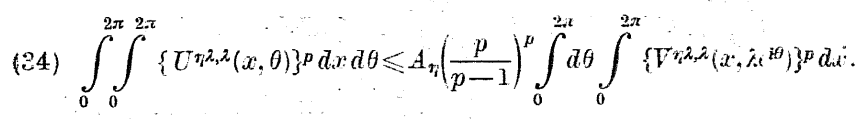

For any fixed $\zeta,|\zeta|<1$, the function $u(\xi, \zeta)$ is subharmonic in $z$, and so

$$
\int_{0}^{2 \pi}\left\{V^{r \lambda, \lambda}\left(x, \lambda \epsilon^{i \theta}\right)\right\}^{p} d x \leqslant A_{n}\left(\frac{p}{p-1}\right)^{p} \int_{0}^{2 \pi} u^{p}\left(\lambda_{t}^{i x}, \lambda e^{i \theta}\right) d u .
$$

Substituting this inequality into the right side of (34). we get (32).

For $p \geqslant 2$ the expression $\{p /(p-1)\}^{p}$ does not exceed 4 . Hence, if we take for $p$ in (32) the value $2 k$, multiply both sides of the inequality by $a^{2 k} /(2 k)$ ! and sum over $k=1,2,3, \ldots$ we obtain

$$
\int_{0}^{2: \tau} \int_{0}^{2 \pi} \cosh \left\{\alpha V^{\eta \lambda, \lambda}(x, \theta)\right\} d x d \theta \leqslant 164_{\eta}^{2} \int_{0}^{2 \pi} \int_{0}^{2 \pi} \cosh \left\{\alpha u\left(\lambda e^{i x}, \lambda e^{i \theta}\right)\right\} d x d \theta
$$


assuming, as we may, that $16 A_{\eta}^{2} \geqslant 1$. Or, on account of the inequality $\frac{1}{2} e^{t} \leqslant \cosh t \leqslant e^{t}$, valid for $t \geqslant 0$,

(35) $\int_{0}^{2 \pi} \int_{0}^{2 \pi} \exp \left\{\alpha D^{\eta \lambda, \lambda}(x, \theta)\right\} d x d \theta \leqslant 32 A_{0}^{2} \int_{0}^{2 \pi} \int_{0}^{2 \pi} \exp \left\{\alpha u\left(\lambda e^{2 x}, \lambda e^{i \theta}\right)\right\} d x d \theta$.

It is now easy to complete the proof of Theorem 4. For let $f(z, \zeta)$ be a function regular for $|z|<1,|\zeta|<1$, and satisfying

$$
\int_{0}^{2 \pi} \int_{0}^{2 \pi}\left|f\left(r e^{i x}, \varrho e^{i \theta}\right)\right|^{\alpha} d x d \theta \leqslant M^{\alpha} \quad(r, \varrho<1, \alpha>0) .
$$

L... Let us now set

$$
\begin{aligned}
u(z, \zeta) & =\log ^{+}|f(z, \zeta)| \\
F^{\eta, \lambda}(x, \theta) & =\operatorname{Max}|f(z, \zeta)| \text { for }(z, \zeta) \in \Delta(x, \lambda \eta, \lambda) \times \Delta(\theta, \lambda \eta, \lambda) \\
F^{\eta}(x, \theta) & =\lim _{2 \rightarrow 1} F^{\eta \lambda, \lambda}(x, \theta) .
\end{aligned}
$$

Then (see (31)),

$$
\begin{aligned}
\int_{0}^{2 \pi} \int_{0}^{2 \pi}\left\{F^{\eta \lambda, \lambda}(x, \theta)\right\}^{\alpha} d x d \theta & \leqslant \int_{0}^{2 \pi} \int_{0}^{2 \pi} \exp \left\{a U^{\eta \lambda, \lambda}(x, \theta)\right\} d x d \theta \\
& \leqslant 32 A_{\eta}^{2} \int_{0}^{2 \pi} \int_{0}^{2 \pi} \exp \left\{a u\left(\lambda e^{i x}, \lambda e^{i \theta}\right)\right\} d x d \theta \\
& \leqslant 32 A_{\eta}^{2} \int_{0}^{2 \pi} \int_{0}^{2 \pi}\left\{\left|f\left(\lambda e^{i x}, \lambda e^{i \theta}\right)\right|^{\alpha}+1\right\} d x d \theta
\end{aligned}
$$

Let us compare the extreme terms here. If we apply the resulting inequality to the function $K f$, where $K$ is a positive constant, and then make $K$ tend to $+\infty$, we get

$$
\int_{0}^{2 \pi} \int_{0}^{2 \pi}\left\{F^{\eta \lambda, \lambda}(x, \theta)\right\}^{\alpha} d x d \theta \leqslant 32 A_{\eta}^{2} \int_{0}^{2 \pi} \int_{0}^{2 \pi}\left|f\left(\lambda e^{i x}, \lambda e^{i \theta}\right)\right|^{\alpha} d x d \theta \leqslant 32 A_{\eta}^{2} M M^{\alpha}
$$

and, making $\lambda \rightarrow 1$,

$$
\int_{0}^{2 \pi} \int_{0}^{2 \pi}\left\{F^{\eta}(x, \theta)\right\}^{\alpha} d x d \theta \leqslant 32 A_{\eta}^{2} M^{\alpha}
$$

which completes the proof of Theorem 4 .
Theorem 5. Under the conditions of Theorem 1, at almost every point $\left(x_{1}, x_{2}, \ldots, x_{k}\right)$ the non-tangential limit

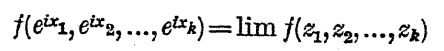

is equal to the iterated limit $f_{x_{1} x_{2} \ldots x_{k}}$.

The proof is almost immediate and it is enough to consider the case $k=2$. Since, by hypothesis, $f\left(e^{i x_{1}}, e^{i x_{2}}\right)$ exists almost everywhere, given any $\varepsilon>0$ we can find a set $E$ of points $\left(x_{1}, x_{2}\right)$ situated in the square $Q\left(0 \leqslant x_{1} \leqslant 2 \pi, 0 \leqslant x_{2} \leqslant 2 \pi\right)$ such that the measure of $Q-E$ is arbitrarily small and that

$$
\begin{gathered}
\left|f\left(r_{1} e^{i x_{1}}, r_{2} e^{i x_{1}}\right)-f\left(e^{i x_{1}}, e^{i x_{2}}\right)\right|<\varepsilon \\
\text { for }\left(x_{1}, x_{2}\right) \in E, 1-r_{1}<\delta, 1-r_{2}<\delta, \delta=\delta(\varepsilon)
\end{gathered}
$$

(we use here the existence of the radial limit only). We know that $f_{x_{1}}\left(z_{2}\right)$ exists for almost every $x_{1}$. Hence, making $r_{1}$ tend to 1 in (37), we see that

$$
\left|f_{x_{1}}\left(r_{2} e^{i x_{2}}\right)-f\left(e^{i x_{1}}, e^{i x_{2}}\right)\right| \leqslant \varepsilon \quad\left(1-r_{2}<\delta\right)
$$

for almost every $x_{1}$ such that $\left(x_{1}, x_{2}\right) \in E$ or, in other words, for almost every $\left(x_{1}, x_{2}\right)$ in $E$. Making now $r_{2}$ tend to 1 we see that

$$
\left|f_{x_{1} x_{2}}-f\left(e^{i x_{1}}, e^{i x_{2}}\right)\right| \leqslant \varepsilon
$$

for almost every point in $E$. Since $\varepsilon$ and the measure of $Q-E$ are arbitrarily small, Theorem 5 is established.

It follows from Theorem 5 that under the assumptions of Theorem 5 (in particular for the functions of the class $H^{\alpha}$ ) the iterated limit of the function $f$ is almost always independent of the order in which the variables $z_{1}, z_{2}, \ldots, z_{k}$ tend successively to limits. It is likely that the result remains valid for the functions $f$ satisfying the conditions of Theorem 2.

Added in proof. 15. IX. 1949. It can be shown that under the assumptions of Theorem 2 the function $f$ tends to a limit as the point $\left(z_{1}, \ldots, z_{k}\right)$ approaches almost every point $\left(e^{i \theta_{1}}, \ldots, e^{i \theta_{k}}\right)$ nontangentially and regularly. "Regularly" means that all the ratios $\left(1-r_{j}\right) /\left(1-r_{k}\right)$ remain bounded. A proof will be given in a paper by A. P. Calderón and myself. 
4. Applications to conjugate series of several variables. First we recall a few familiar facts from the theory of trigonometric series ${ }^{12}$ )

$$
\frac{1}{2} a_{0}+\sum_{n=1}^{\infty}\left(a_{n} \cos n x+b_{n} \sin n x\right)
$$

of a single variable (we restrict ourselves to series with real coefficients). This series is the real part of the power series

$$
\frac{1}{2} a_{0}+\sum_{n=1}^{\infty}\left(a_{n}-i b_{n}\right) 2^{n}
$$

if we set here $z=t^{i x}$. The imaginary part of $(39)$, under the same assumption, is

$$
\sum_{n=1}^{\infty}\left(a_{n} \sin n x-b_{n} \cos n x\right)
$$

and is called the conjugate series of (39). If we denote (39) by $\mathscr{B}$, its conjugate will be denoted by $\widetilde{S}$. If $S$ is the Fourier series of a (Lebesgue) integrable function $f$, then almost everywhere $\widetilde{s}$ is summable Abel (or even $(C, 1)$ ) to sum

$$
\tilde{f}(x)=-\frac{1}{\pi} \int_{-\pi}^{\pi} f(x+t) \frac{1}{2} \cot \frac{1}{2} t d t=-\frac{1}{\pi} \lim _{x \rightarrow+0} \int_{-\pi}^{(\varepsilon) \pi}
$$

where $\int_{-\pi}^{(\varepsilon) \pi}$ denotes the integral extended over the part of $(-\pi, \pi)$ situated outside the interval $(-\varepsilon, \varepsilon)$. The result includes the assertion that $\tilde{f}(x)$ exists almost everywhere for every integrable $f$.

$$
\text { If we write } S \text { in the form }
$$

$$
\sum_{n=-\infty}^{+\infty} e_{n} e^{i n x}, \quad e_{n}=\frac{1}{2}\left(a_{n}-i b_{n}\right), n \geqslant 0, a_{-n}=\tilde{c}_{\dot{n}},
$$

then $\widetilde{\mathbb{S}}$ can be written

$$
\sum_{n=-\infty}^{+\infty}(-i \operatorname{sign} n) c_{n} e^{i n x}
$$

12) All the results needed here will be found in $T S$, Ch. VII. For the inequality (45), see TS, p. 165 . Ex. 7 .
The very well known result of $M$. Ries z asserts that if $f$ belongs to $L^{p}, p>1$, so does $\widetilde{f}$. In what follows we shall also need the following two inequalities

$$
\begin{gathered}
\left(\int_{-\pi}^{\pi}|\tilde{f}|^{\delta} d a\right)^{1 / \delta} \leqslant A_{\delta} \int_{-\pi}^{\pi}|f| d x \\
\int_{-\pi}^{\pi}|\tilde{f}|(\log +\tilde{g} \mid)^{\alpha-1} d x \leqslant A_{\alpha} \int_{-\pi}^{\pi}|f|(\log +|f|)^{\alpha} d x+A_{\alpha}
\end{gathered}
$$

the latter especially in the case $\alpha=1$.

Let us now consider a double trigonometrie series

$$
\sum_{m, n=-\infty}^{+\infty} c_{m n} \epsilon^{i(m x+n y)} \quad e_{-m,-n}=\bar{c}_{m, n-}
$$

It can be written in the equivalent form

$$
\begin{aligned}
& \sum_{m, n=0}^{\infty}\left(\alpha_{m n} \cos m x \cos n y+\beta_{m n} \sin m x \cos n y+\right. \\
& \left.\quad+\gamma_{m n} \cos m x \sin n y+\delta_{m n} \sin m x \sin n y\right) \lambda_{m n},
\end{aligned}
$$

where the multipliers $\lambda_{m n}$ are introduced for the sake convenience only and are defined by the equations

$$
\hat{\lambda}_{m n}=1, \quad \hat{\lambda}_{m 0}=\hat{\lambda}_{0 n}=\frac{1}{2}, \quad \hat{\lambda}_{00}=\frac{1}{2}
$$

The coefficients $\alpha, \beta, \gamma, \delta$ are then easily expressible in terms of the $c_{m n}$. For example, $a_{m n}=c_{m, n}+c_{m,-n}+c_{-m, n}+c_{-m,-n}$, etc. If $c_{m n}=0$ whenever $m n=0$, we shall say that (46), or (47), is of restricted type.

Let us now consider a power series

$$
\sum_{m, n=0}^{\infty}\left(A_{m n}-i B_{m n}\right) z^{m} \zeta^{n}
$$

of two variables. The real part of it for $z=t^{i x}, \zeta=t^{t y}$ is

$$
\sum_{m, n=0}^{\infty} A_{m n} \cos (m x+n y)+B_{m n} \sin (m x+n y)
$$

which shows that only special series (47) are real parts of power series of two variables. Writirg (49) in the form (46), we see that a necessary and suffieient condition for (46) to be the real part of a power 
series is that $c_{m n}=0$ for $m n<0$. If (49) is the real a part of (48), the imaginary part is

$$
\sum_{m, n=0}^{\infty} A_{m n} \sin (m x+n y)-B_{m n} \cos (m x+n y) .
$$

Thus the notion of conjugate series does not appear naturally in the case of series of two variables. We may however introduce: it formally. For using the definition (43) we may consider alongside. (46) the three series

$$
\sum c_{m n}(-i \operatorname{sign} m) e^{i(m x+n y)}, \quad \sum c_{m n}(-i \operatorname{sign} n) \epsilon^{i(m x+n y)}
$$

$$
\sum c_{m n}(\text {-sign } m n) c^{i(m x+n y)}
$$

which will be the conjugates of $S$ with respect to $x$, to $y$, and to $x, y$. If we denote $(46)$ by $S$, these series will be written $\widetilde{S}^{x}, \widetilde{S^{y}}$ and $\widetilde{S}^{x y y}$ respectively. The series $\widetilde{S}^{x y}=\widetilde{S}^{y x}$ is always of restricted type. If $S$ is of restricted type and of the form (49), then (50) is both $\widetilde{\mathbb{S}^{x}}$ and $\widetilde{\mathbb{S}^{y}}$. The series (50) will be called the conjugate of (49). If the constant. term of the power series (48) is real, an assumption which we can always make, the constant term of $(50)$ is zero.

It is important to observe that, for the most general series $\mathbb{S}$ in (48), $S-\widetilde{S}^{x y}$ is always the real part of a power series. If, in addition, $\boldsymbol{S}$ is of restricted type then the conjugate of $S-\widetilde{S}^{x} y$ is $\widetilde{S^{x}}+\widetilde{S^{x}}$. For

$$
S-\widetilde{S^{x}}=\sum c_{m n}(1+\operatorname{sign} m n) \epsilon^{\ell(m x+n y)}
$$

and the coefficients on the right are zero if $m n<0$. The second part of the italicized statement follows immediately from the formula:

$$
(1+\operatorname{sign} m n) \operatorname{sign} m=\operatorname{sign} m+\operatorname{sign} n \quad(m, n \neq 0) .
$$

The importance of this lies in the fact that though (unlike in the case of series of one variable) the complex methods cannot be applied to the general series $S$, they are applicable to $S-\widetilde{S}^{x y}$. If we can obtain some information about the behavior of the latter series, and if we know the behavior of $S$, we get information about $\widetilde{S^{x p}}$.

As an application of this idea we are going to prove the following result, in which $f$ is of period $2 \pi$ with respect to $x$ and $y$, and $Q$ denotes the square $|x| \leqslant \pi,|y| \leqslant \pi$.
Theorem 6'. If $f(x, y) \log +|f(x, y)| \in I$, then

$$
\begin{gathered}
\tilde{f}(x, y)=\frac{1}{\pi^{2}} \int_{-\pi}^{\pi} \int_{-\pi}^{\pi} f(x+u, y+v) \frac{1}{2} \cot \frac{1}{2} u \frac{1}{2} \cot \frac{1}{2} v d u d v= \\
=\frac{1}{\pi^{2}} \lim _{\varepsilon, \eta \rightarrow+0} \int_{-\pi}^{(e) \pi} \int_{-\pi}^{(\eta) \pi}
\end{gathered}
$$

exists almost everywhere. Moreover

$$
\left(\iint_{Q}\left[\left.\tilde{f}\right|^{\delta} d x d y\right)^{1 / \delta} \leqslant A_{\delta} \iint_{Q}|f| \log +|f| d x d y+A_{\delta}\right.
$$

The existence of $\tilde{f}$ for $f \in L^{p}, p>1$, was proved by Sok 6 Sokolowski [1]. He even showed that $\tilde{f}$ exists if $f(\log +|f|)^{3}$ is integrable, and he conjectured that this result holds if the exponent 3 is replaced by 1 .

In proving Theorem $6^{\prime}$ we shall be concise and we shall omit certain details which can be found in the paper just quoted. Let us denote the $(C, 1,1)$ means of the series $(51)$ respectively by $\widetilde{\sigma}_{m n}^{x}(x, y)$, $\tilde{\sigma}_{m n}^{g}(x, y), \widetilde{\sigma}_{m n}^{x y}(x, y)$, and the Abel means by $\widetilde{P}_{r e}^{x}(x, y), \widetilde{P}_{r e}^{g}(x, y)$, $\widetilde{P}_{r e}^{x y}(x, y)$. Sokolowski proves (1. c., Theorem 4) that, under the assumptions of Theorem $6^{\prime}$,

$$
\begin{gathered}
\tilde{\sigma}_{m n}^{x g}(x, y)-\frac{1}{\pi^{2}} \int_{-\pi}^{(\varepsilon) \pi} \int_{-\pi}^{(\eta) \pi} f(x+u, y+v) \frac{1}{2} \cot \frac{1}{2} u \frac{1}{2} \cot \frac{1}{2} v d u d v \rightarrow 0 \\
\left(\varepsilon, \eta \rightarrow 0, m=\left[\frac{1}{\varepsilon}\right], n=\left[\frac{1}{\eta}\right]\right)
\end{gathered}
$$

almost everywhere, and exactly the same proof (for Fejér's and Poisson's kernels satisfy similar inequalities) shows that

$$
(\varepsilon=1-r \rightarrow 0, \eta=1-\varrho \rightarrow 0) \text {. }
$$

Let us assume first that $S$, defined by (46), is of restricted type. Since $f \log ^{+}|f|$ is integrable, both $\widetilde{S}^{x}$ and $\widetilde{S}^{y}$ are Fourier series of integrable functions $\left.\widetilde{f^{x}} x, y\right)$ and $\widetilde{f^{y}}(x, y)$, and so $\nabla=\widetilde{S}^{x}+\widetilde{S}^{y}$ is the Fourier series of $h=\widetilde{f^{x}}+\widetilde{f}^{y} \varepsilon L$. Moreover $\nabla$ is the imaginary part, 
on the unit circle, of a (restricted) power series $F(z, \zeta)$ defiried by (48). Hence the real part $U=S-\widetilde{S^{x} y}=\sum u_{m n} \iota^{(i(m x+n y)}$ of that power series is of the class $L^{\delta}$, for every $0<\delta<1$. More precisely, if we set $u_{r e}(x, y)=\sum u_{m n} \gamma^{|m|} l_{\ell}|n|_{c}^{i(m x+n y)}$, then

$$
\begin{aligned}
\left(\iint_{Q}\left|u_{r e}(x, y)\right|^{\delta} d x d y\right)^{1 / \delta} & \leqslant A_{\delta} \iint_{Q}|\hbar(x, y)| d x d y \\
& \leqslant A_{\delta} \iint_{Q}|f| \log ^{+}|f| d x d y+A_{\delta} .
\end{aligned}
$$

It follows that $F(z, \zeta) \in H^{\delta}$ for every: $\delta<1$, and so, on account of Theorem 1, has a non-tangential limit almost everywhere. In particular, $S-\widetilde{S}^{x y}$ is Abel summable at almost every point. Since the same holds for $S$ (see Jessen, Marcinkiewicz and Zygmund [1]), $\widetilde{\delta}^{x y}$ is Abei summable almost everywhere. On account of (54), this completes the proof of the first part of Theorem $6^{\prime}$, under the assumptions that $S$ is of restricted type.

To dispose of that assumption we consider the function

$$
g_{(x, y)}=\frac{1}{2 \pi} \int_{-\pi}^{\pi} f(x, y) d x+\frac{1}{2 \pi} \int_{-\pi}^{\pi} f(x, y) d y-\frac{1}{ \pm \pi^{2}} \int_{-\pi}^{\pi} \int_{-\pi}^{\pi} f(x, y) d x d y ;
$$

which has the same "marginal" coefficients $c_{m 0}, c_{0 n}$ as $f$, the remaining coefficients being all equal to zero. Thus the Fourier series of $f_{1}=f-g$ is of restricted type, and since $g \log ^{+}|g| \epsilon L$ the function $f_{1} \log ^{+}\left|f_{1}\right|$ is integrable, so that $\tilde{j}_{1}^{x y}(x, y)$ exists almost everywhere. On the other hand, $\widetilde{g}^{x y}(x, y)$ exists everywhere and vanishes identically. Hence $\tilde{\gamma}^{x y}$ exi its almost everywhere.

The second part of Theorem $6^{\prime}$ follows immediately from (55), and the extension to "non-restricted" series is again achieved by considering the function (56).

The purpose of the foregoing argument was primarily to show how complex methods can be applied to double trigonometric series, and we are not interested in exploiting the field of conjugate series here. We may however state without proof some additional result which can easily be proved.

First of all, from Theorem $6^{\prime}$ and from (53) follows that $\widetilde{S}^{x_{x}}$ is almost everywhere summable by the method $(C, 1,1)$ (or even summable $(C, \alpha, \beta), \alpha>0, \beta>0$, as can be shown similarly). We have already stated the fact that $\widetilde{S^{x y}}$ is almost everywhere summable
Abel. That $\tilde{\mathcal{Y}}_{r e}^{x y}(x, y)$ has non-tangential limits almost everywhere can ke proved similarly. An application of (15) shows that

$\iint_{Q}\left[\tilde{f} x y\left|\{\log (2+\tilde{\Gamma}, x y \mid)\}^{\alpha-2} d x d y \leqslant A_{a} \iint_{Q}\right| f|\log +|\right\},{ }^{\alpha} d x d y+A_{a} \quad(a>1)$,

an inequality which for $a=1$ degenerates into the second part of Theorem $6^{\prime}$. What is more, both inequalities hold if instead of $\tilde{j}^{x g}$ we write there

$$
\begin{aligned}
& \tilde{f}^{*}(n, y)=\sup _{\varepsilon, \tau_{\pi}}\left|\pi^{-2} \int_{-\pi}^{(\varepsilon) \pi} \int_{-\pi}^{\pi} f(x+u, y+v) \frac{1}{2} \cot \frac{1}{2} u \frac{1}{2} \cot \frac{1}{2} x d u d v\right|(\varepsilon<\pi, \eta<\pi) \\
& \text { or } \widetilde{\sigma}^{*}(x, y)=\sup _{m, n}\left|\sim_{m, n}^{x y}(x, y)\right| \text {, or } \widetilde{P}^{*}(x, y)=\operatorname{Sup}_{r e}\left|\widetilde{F}_{r e}^{x y}(x, y)\right| \text {. }
\end{aligned}
$$

We end by a few remarks concerning trigonometric series is of $k$ variables

$$
\sum_{n_{1}, \ldots, n_{k}=-\infty}^{+\infty} c_{n_{1} \ldots n_{k}} e^{l\left(n_{1} x_{1}+\ldots+n_{k} x_{k}\right)}
$$

The notions of restricted series and of conjugate series are define: as before. A necessary and sufficient condition for $S$ to be the real part of a power series $\sum c_{n_{1} \ldots n_{k}} z_{1}^{n_{1} \ldots z^{n_{k}}}$ is that all the coefficients $c_{n_{1} \ldots n_{k}}$ whose indices distinct from 0 are not of the same sign vanish.

Theorem 6. Let $f\left(x_{1}, \ldots, x_{k}\right)$ be of period $2 \pi$ with respect to each variable and let $f\left(\left.\log ^{+}|j|\right|^{k-1} \in L\right.$. Then

$\widetilde{f}\left(x_{1}, \ldots, x_{k}\right)=$

$=\lim _{\varepsilon_{1} \ldots \varepsilon_{k} \rightarrow 0} \frac{(-1)^{k}}{\pi^{k}} \int_{-\pi}^{\left(\varepsilon_{1}\right) \pi} \ldots \int_{-\pi}^{\left(\varepsilon_{k}\right) \pi} f\left(x_{1}+u_{1}, \ldots, x_{k}+u_{k}\right)_{\frac{1}{2} 2} \cot \frac{1}{2} u_{1} \ldots \frac{1}{2} \cot \frac{1}{2} u_{k} d u_{1} \ldots d u_{k}$ exists almost exerywhere, and

$$
\left(\left.\int \underset{Q_{k}}{\cdots} \int \tilde{\mid}\right|^{j} d x_{1} \ldots d x_{k}\right)^{1 / \delta} \leqslant A_{\tilde{\delta}, k} \int_{Q_{k}} \cdots \int|f|\left(\log +\left.||\right|^{k-1} d x_{1} \ldots d x_{k}+A_{\tilde{\delta}, k} .\right.
$$

If we use (44) and (45) the proof of Theorem 6 follows the same line as that of Theorem 6 , provided we show that a proper linear eombination of $S$ and of its conjugates is always the real part of a power series. We confine our attention to this problem only. 
Let $S$ be an arbitrary series (57). Let $\sum S^{x_{1}}$ denote the sum of the conjugates of $S$ with respect to one variable, $\sum S^{x_{1} x_{2}}$ - the sum of the conjugates with respect to two distinct variables, etc. We shall show that

$$
S-\sum S^{x_{1} x_{2}}+\sum S^{x_{1} x_{2} x_{0} x_{2}}-\ldots
$$

is always the real part of a power series of $k$ variables, and that if $s$ is of restricted type, the imaginary part of that power series will be.

$$
\sum S^{x_{1}}-\sum S^{x_{1} x_{2} x_{4}}+\cdots
$$

For let $n_{1}, n_{2}, \ldots, n_{k}$ be any set of indices all distinct from zero, and let $\eta_{j}=\operatorname{sign} n_{j}$. We have to show that if the $n_{j}$ are not of the same sign then

$$
\text { (60) } 1+\sum \eta_{1} \eta_{2}+\sum \eta_{1} \eta_{2} \eta_{3} \eta_{4}+
$$

is zero. For let us assume for the sake of definiteness that $\eta_{0}$ is even (the proof for $k$ odd is similar) and let us denote the sum (60) by $P_{\boldsymbol{k}}$. Let us denote a similar sum formed with $\eta_{1}, \ldots, \eta_{k-2}$ by $P_{k-2}$ and let the sum

$$
\sum \eta_{1}+\sum \eta_{1} \eta_{2} \eta_{3}+\cdots
$$

be $N_{k-2}$. Then one easily verifies the relation $P_{k}=P_{k-2}\left(1+\eta_{k-1} \eta_{k}\right)+$ $+N_{k-2}\left(\eta_{k-1}+\eta_{k}\right)$, from which immediately follows that $P_{k}=0$ if for example $\eta_{k-1} \eta_{k}<0$.

If $S$ is of resticted type, so is (58), and one easily verifies that the conjugate of (58) with respect, say, to $x_{1}$ is (59).

The other remarks we made about double trigonometric series. are immediately extensible to the series (57).

\section{Buenos Aires, R. Argentina 1. VI. 1948.}

\section{References}

Bergman S. [1], Über ausgezeichnete Randflächen in der Theorie der analytischen Funktionen von zwei komplexen Veränderlichen, Math. Annalen 104 (1931), pp. 611-636.

- [2], On a generalized Green's function and certain of its applications, Bull. American Math. Soc. 47 (1941), pp. 651-661.

Bergman S. and Marcinkiewicz J. [1], Sur les fonctions analytiques de deux variables complexes, Fundamenta Mathematicae 33 (1939-1946), pp. 75-94.

Bers L. [1], On bounded analytic functions of two complex variables in certain domains with distinguished boundary surfaces, American Journal of Math. 64 (1942), pp. 514-529.
Bochner S. [1], Boundary values of analytic functions in several variables and of alnost periodic functions, Annals of Mathematics 45 (1944), pp. 708-722. Cotlar M. and Vignaux J. C. [1], Totally normal families of holomorphic functions (Spanish), Publ. Inst. Mat. Univ. Nac. Littoral 7 (1944), pp. 152. Hardy G. H. and Littlewood J. E. [1], A maximal theorem with functionthearetic applications, Acta Math. 54 (1930), pp. 81-116.

Jessen B., Marcinkiewiez J. and Zygmund A. [1], Note on the differentiability of multiple integrals, Fundamenta Mathematicae 25 (1935), pp. 217-234 Littlew ood J. E. [1], On functions subharmonic in a circle (II), Proc. London Math. Soc. 28 (1928), pp. 383-394.

Paley R. E. A. C. and Zygmund A. [1], A note on analytic functiona inside the unit circle, Proc. Cambridge Phil. Soc. 28 (1932), pp. 266-272. Saks S. [1], Theory of Integral, Warsaw 1937.

Sokół-Sobołowski K. [1], On trigonometric series conjugate to Fourier series of two variables, Fund. Math. 33 (1939-1946), pp. 166-182.

Zygmund A. [1], Trigonometrical Series, Monogr. Mat. V, Warsaw, 1935. - [2], On the differentiability of multiple integrals, Fund. Matb. 23 (1934), pp. 143-155. 